\title{
FATIGUE IN SYSTEMIC LUPUS ERYTHEMATOSUS AND OTHER AUTOIMMUNE SKIN DISEASES
}

\author{
M. Tarazi ${ }^{\# 1,2,3}$, R. G. Gaffney ${ }^{\# 1,2,4}$, D. Pearson ${ }^{1,2}$, C. J. Kushner ${ }^{1,2,5}$, and V. P. Werth ${ }^{1,2}$ \\ ${ }^{1}$ Department of Dermatology, University of Pennsylvania, Philadelphia, PA. \\ ${ }^{2}$ Corporal Michael J. Crescenz VAMC, Philadelphia, PA \\ ${ }^{3}$ Sidney Kimmel Medical College, Thomas Jefferson University, Philadelphia, PA. \\ ${ }^{4}$ Rutgers Robert Wood Johnson Medical School, New Brunswick, NJ. \\ ${ }^{5}$ Perelman School of Medicine, University of Pennsylvania, Philadelphia, PA. \\ \# These authors contributed equally to this work.
}

\section{Abstract}

Background: Fatigue is a well-established symptom in systemic lupus erythematosus (SLE), but has not been well-characterized in other skin-limited autoimmune diseases such as cutaneous lupus erythematosus (CLE), amyopathic dermatomyositis (ADM), or autoimmune blistering diseases (AIBD).

Objectives: In this retrospective study, we compared fatigue in controls $(n=84)$ to patients enrolled in prospective longitudinal databases with SLE ( $n=165)$, CLE $(n=226)$ ADM $(n=136)$, and $\operatorname{AIBD}(\mathrm{n}=79)$.

Methods: We used the Short-Form 36 (SF-36) vitality scale to analyze median scores and percentage of patients with clinically significant fatigue (defined as a score $\mathbf{3 5}$ ) between experimental groups and controls.

Results: Median vitality scores demonstrated greater fatigue in experimental groups ( $\mathrm{SLE}=35$, $\mathrm{IQR}=20-55 ; \mathrm{CLE}=50, \mathrm{IQR}=30-70 ; \mathrm{ADM}=50, \mathrm{IQR}=30-65 ; \mathrm{AIBD}=55, \mathrm{IQR}=35-70$ ) than controls (73, IQR=65-85) $(\mathrm{p}<0.05$ between each experimental group vs. control). The SLE group had worse fatigue than all other groups ( $p<0.05$ SLE vs. each group), but there was no difference between CLE ( $p>0.05)$, ADM ( $p>0.05$ ), or AIBD ( $p>0.05$ ). In addition, experimental groups had more clinically significant fatigue (score 35 ) (SLE, 44.2\%; CLE, 25.2\%; ADM, 31.6\%; AIBD, $24.1 \%)$ than controls $(2 \%)(\mathrm{p}<0.01$ between each experimental group vs. control). The SLE group had more clinically significant fatigue compared to CLE ( $p<0.01)$, however, there was no difference in clinically significant fatigue between SLE versus ADM $(\mathrm{p}=0.17)$ or AIBD $(\mathrm{p}=0.055)$.

Conclusions: These findings demonstrate that patients with skin-limited autoimmune disease experience more fatigue than controls. Fatigue is an important symptom that negatively affects

Correspondence: Victoria P. Werth, MD, Department of Dermatology, Perelman Center for Advanced Medicine, Suite 1-330A, 3400 Civic Center Boulevard, Philadelphia, PA 19104, USA., Tel: +1 215823 4208, fax: +1 866755 0625,

werth@pennmedicine.upenn.edu.

Disclosures: None Declared. 
quality of life for patients and should be addressed by clinicians and measured in future clinical trials.

\section{INTRODUCTION}

Fatigue is a well-established symptom in systemic lupus erythematosus (SLE) that significantly impacts patients' quality of life (QOL) and contributes to disease morbidity. It has been described as an abnormal or extreme whole body tiredness, that is disproportionate to activity or exertion [1]. 53\% to $80 \%$ of SLE patients identified fatigue as one of their primary symptoms and its etiology is thought to be multifactorial [1]. Studies aimed at finding a relationship between fatigue and immunologic, inflammatory, or other disease characteristics have shown inconsistent results. Fatigue strongly correlates with psychosocial factors such as mood disorders, anxiety, poor sleep quality, and chronic pain syndromes [2].

Although patients with skin-limited autoimmune diseases anecdotally report significant fatigue, there is limited data in patients with conditions such as cutaneous lupus erythematosus (CLE), amyopathic dermatomyositis (ADM), and autoimmune blistering diseases (AIBD). The aim of this study is to compare fatigue severity between these skinlimited autoimmune conditions and SLE, as well as to healthy control groups. Given what is known about fatigue's influence on QOL in multiple different inflammatory conditions, it is important to gain a better understanding of the prevalence of fatigue in patients with skinlimited autoimmune diseases. In this retrospective study, we use median SF-36 vitality scores to compare fatigue in historic controls to patients enrolled in prospective longitudinal databases with SLE, CLE, ADM and AIBD. We also examined the percentage of patients in each group who experience "clinically significant" fatigue.

\section{METHODS}

\section{Patients}

This study was reviewed and approved by the Institutional Review Board at the University of Pennsylvania. The study population included patients enrolled in prospective longitudinal databases with SLE ( $n=165), \operatorname{CLE}(n=226), \operatorname{ADM}(n=136)$, and AIBD $(n=79)$. The AIBD database included patients with immunologic or histologically proven bullous pemphigoid, pemphigus vulgaris, and pemphigus foliaceus. Historic controls $(n=84)$ were selected from a study performed by Skoie et al. that examined fatigue in psoriasis and consisted of healthy acquaintances of psoriasis patients from Stavanger University Hospital in Norway [3]. These patients were Norwegian speaking and age 18 or older; exclusion criteria in Skoie et al's study included a diagnosis of psoriasis, prior history of cancer, chronic inflammatory diseases, or untreated hyper or hypothyroidism. We collected baseline characteristics including age, gender, smoking status, comorbidities, autoimmune skin disease-related medications in the study population, and disease severity. Disease severity was reported using the following validated measurement instruments: Cutaneous Lupus Erythematosus Disease Area and Severity Index (CLASI), Cutaneous Dermatomyositis Disease Area and Severity Index (CDASI), and the Bullous/Pemphigus Disease Area Index (B/PDAI). For historic controls, information was not complete regarding certain comorbidities or medications. 


\section{Measurement of Fatigue}

The Short-Form 36 (SF-36) is a 36-item patient-reported survey that measures different health status through the following subscales: physical functioning, role limitations to physical problems, social functioning, bodily pain, general mental health, role limitations due to emotional problems, vitality, and general health perceptions. Scores are valued between 0 and 100, with a higher score defining a more favorable health state. Fatigue severity was assessed with the vitality subscale and general mental health was assessed with the mental health subscale using patients' scores recorded from their first visit [4]. Clinically significant fatigue was defined as a score $₫ 5[3,5]$. This cut-off point was used by Dagfinrud et al in their investigation of fatigue in patients with ankylosing spondylitis [5]. They assigned vitality scores below the $10^{\text {th }}$ percentile of the general population to the "low vitality" group, suggesting that "clinically significant" fatigue occurs in $10 \%$ of the general population.

\section{Statistical Analysis}

We compared the median SF-36 vitality scores between our skin disease groups and the control group using the Kruskal-Wallis and post-hoc Dunn's multiple comparison tests. We also compared the percentage of subjects with clinically significant fatigue (score $\mathbf{3 5}$ ) in each group through the Fisher's exact test. Using the Bonferoni correction for multiple comparisons, $\mathrm{P}$ values $<0.005$ were considered statistically significant.

Univariate and multivariate logistic regression analyses were performed comparing the presence of clinically significant fatigue and covariates such as age, sex, smoking status, comorbidities, medications, and SF-36 mental health score (Table 2). Variables with P values $<0.25$ in the univariate analysis were retained in the multivariable model for SLE, CLE, $\mathrm{ADM}$, and AIBD. In the final model, $\mathrm{P}$ values $<0.05$ were considered statistically significant (Table 3).

\section{RESULTS}

\section{Fatigue Severity between Groups}

The majority of our autoimmune skin disease groups were made up of females and the mean ages were $45 \pm 13.96,49 \pm 14.60,48 \pm 13.00,55 \pm 12.95$, and $65 \pm 16.10$ in controls, CLE, SLE, ADM, and AIBD, respectively. The average CLASI, CDASI, and B/PDAI scores were 9.9 for SLE, 9.7 for CLE, 19.4 for ADM, and 9.6 for AIBD. The remaining baseline characteristics of the control and autoimmune skin disease groups are presented in Table 1. Autoimmune skin disease patients demonstrated greater fatigue measured by median vitality scores $(\mathrm{SLE}=35, \mathrm{IQR}=20-55 ; \mathrm{CLE}=50, \mathrm{IQR}=30-70 ; \mathrm{ADM}=50, \mathrm{IQR}=30-65 ; \mathrm{AIBD}=55$, $\mathrm{IQR}=35-70)$ compared to controls $(73, \mathrm{IQR}=65-85)(\mathrm{p}<0.05$ between SLE, CLE, ADM, and AIBD groups vs. control) (Figure 1). Among autoimmune skin disease patients, the SLE group had significantly worse fatigue compared to all other groups ( $\mathrm{p}<0.05$ SLE vs. CLE, $\mathrm{ADM}$, and AIBD). There was no difference in median vitality scores between the CLE $(\mathrm{p}>0.05), A D M(p>0.05)$, or AIBD groups ( $>>0.05)$. After examining median vitality scores, we then assessed which groups had more patients with clinically significant fatigue (score $\leq$ 35 ). We found that autoimmune skin disease groups had more clinically significant fatigue 
(SLE, 73/165 [44.2\%]; CLE, 57/226 [25.2\%]; ADM, 43/136 [31.6\%]; AIBD, 19/79 [24.1\%]) than controls $(2 / 84[2 \%])(\mathrm{p}<0.01$ between each experimental group vs. control) (Figure 2). Among autoimmune skin disease patients, the SLE group had more clinically significant fatigue compared to $\operatorname{CLE}(\mathrm{p}<0.01)$. However, there was no difference in clinically significant fatigue between SLE versus ADM $(\mathrm{p}=0.17)$ or AIBD $(\mathrm{p}=0.06)$.

\section{Factors Associated with Fatigue}

We examined potential influential factors on fatigue through a multivariate logistic regression for each autoimmune skin disease group (Table 3). In all autoimmune skin disease groups, patients with higher mental health scores were less likely to have clinically significant fatigue (Table 3). CLE patients taking immunosuppressant medications were more likely to have clinically significant fatigue (OR: 2.62; 95\% CI: 1.20 to 5.74; p>0.016), however, this association was not seen in the other autoimmune skin disease groups. The multivariable logistic regression revealed that female SLE patients and ADM patients with hypothyroid disease were more likely to have clinically significant fatigue [(OR: 6.45; $95 \%$ CI: 1.20 to $5.74 ; p>0.016$ ) and (OR: $4.21 ; 95 \%$ CI: 1.31 to $13.52 ; p>0.016)$ respectively] compared to patients without these covariables.

\section{DISCUSSION}

In this study, we examined the prevalence of fatigue in SLE, CLE, ADM, and AIBD compared to healthy controls. Our results demonstrate that skin-limited autoimmune patients have significantly worse fatigue than healthy controls. When examining fatigue between the autoimmune skin disease groups, we found that SLE patients had worse fatigue scores than CLE, ADM, and AIBD, which is expected given the systemic nature of the disease. Surprisingly, however, there was no significant difference in the prevalence of clinically significant fatigue between SLE and ADM or AIBD. While patients with SLE had worse fatigue scores overall, there was a similar prevalence of clinically significant fatigue in patients with cutaneous-limited disease. This finding has critical implications for future clinical trials, which should consider fatigue outcomes when assessing the efficacy of a new therapy.

The second goal of this study was to identify and quantify associations between clinical variables and fatigue using a multivariate model. The variables identified are inconsistent between SLE, CLE, ADM, and AIBD, which is likely due to the demographic heterogeneity between the groups and relatively small sample size. However, the influential variables found in this study are still of clinical importance and should be explored to better understand fatigue in this population. Furthermore, these variables can help physicians to identify and counsel patients who are more likely to experience clinically significant fatigue in their clinic.

Across all autoimmune skin disease patients, fatigue was influenced by mental health score, which we used as a surrogate for depressed mood and emotional well-being [6]. Postulated mechanisms propose that chronic inflammatory states can lead to cytokine-induced "sickness behavior," which shares many features with depression, such as fatigue and anhedonia. There is also evidence to suggest that major depression is associated with pro- 
inflammatory cytokines, and this relationship may therefore be bi-directional [7-9]. This finding is further supported by clinical studies in SLE that have shown a relationship between fatigue and mood disorders [2].

The association between immunosuppressants and fatigue was only seen in CLE and can be explained by the treatment algorithm for the disease. CLE patients are placed on immunosuppressants when their disease is severe with widespread skin manifestations and is refractory to topical steroids and antimalarials $[10,11]$. Though disease activity was not examined in the multivariate analysis, CLE patients on immunosuppressants may have more debilitating disease that could be contributing to their fatigue. If on the other hand, the medications contribute to patients' fatigue, it is important for clinical trials to consider fatigue as an outcome measure when assessing new treatment options.

The fatigue-associated variables such as thyroid disease in ADM and female gender in SLE are consistent with past studies. Fatigue is a hallmark symptom in thyroid disease and autoimmune thyroid dysfunction is particularly common in dermatomyositis [12, 13]. Fatigue has also been found to be more prevalent in women [14].

The results of this study suggest that having an autoimmune skin condition significantly contributes to fatigue. This finding has been seen in other inflammatory diseases such as ankylosing spondylitis (AS) and psoriasis [3, 5]. A study performed by Dagfinrud et al showed that a median of $30 \%$ of patients with AS experienced clinically significant fatigue compared to $10 \%$ of the general population [5]. Though patients with AS have significant limitation of movement and pain, which could be contributing to their fatigue, the percentage of patients with clinically significant fatigue is comparable to those seen in our autoimmune skin disease population. Similarly, Skoie at al examined fatigue in psoriasis and found that $42 \%$ of patients experienced clinically significant fatigue as opposed to $2 \%$ of healthy controls [3]. As mentioned earlier, pro-inflammatory cytokines generated by the innate immune system may cross the blood brain barrier and generate an adaptive response, termed "sickness behavior," which acts to conserve energy and cause fatigue $[3,7,15]$.

This study had several limitations. It is a retrospective study of prospectively collected data at a single institution comparing autoimmune skin disease patients to a historic control. Our groups were not matched by age and sex which impacts the internal validity of the study; however, these variables were included in the multivariate analysis and their effects are included in tables 2 and 3. The control group consisted of healthy controls living in Norway which has a predominantly Caucasian population. Although DM usually presents in Caucasian females, SLE/CLE is a more racially heterogeneous group. These inherent differences were not controlled for in this study. Additionally, our control group was not included in our multivariate analysis due to missing data on thyroid disease in this group, as well as information on their mental health scores. Another limitation is that the study was conducted at a quaternary referral center, and patients included may have more refractory or severe disease. Studies examining the relationship between fatigue and disease activity in patients with SLE have failed to demonstrate a conclusive association between these two factors [1]. However, patients' interpretation of more severe disease status and feelings of hopelessness towards their condition may contribute to greater fatigue. Finally, fatigue is a 
multifactorial symptom and therefore all contributing factors, such as employment type and socioeconomic status, were not controlled for or evaluated.

In conclusion, the results of this study indicate that fatigue is more prevalent in SLE, CLE, $\mathrm{ADM}$ and AIBD compared to healthy controls. Interestingly, we found that patients with skin-limited disease, such as ADM and AIBD, had the same prevalence of clinically significant fatigue as SLE, while patients with CLE have less fatigue. Fatigue is a debilitating symptom that significantly contributes to QOL in patients with inflammatory conditions. It is important for physicians to recognize and address this symptom in patients with both skin-limited and systemic autoimmune diseases, and perhaps refer for counseling or offer interventions for fatigue and coexisting mood disorders. Moreover, fatigue should be further investigated in these conditions to determine its effect on patients' QOL and its potential as an outcome measure in clinical trials for skin-limited autoimmune diseases.

\section{Acknowledgements}

The authors would like to thank Dr. Inger Marie Skoie and Dr. Roald Omdal of Stavanger University Hospital, Stavanger, Norway for generously sharing their data.

This work was supported by the United States Department of Veterans Affairs (Veterans Health Administration, Office of Research and Development and Biomedical Laboratory Research and Development) Merit Review 5 I01 BX000706-04 (PI: Werth) and by the United States Department of Health and Human Services and the National Institutes of Health (National Institute of Arthritis and Musculoskeletal and Skin Diseases) R21 AR066286 (PI: Werth).

Funding Statement: No sponsorship.

\section{References}

1. Ahn GE and Ramsey-Goldman R, Fatigue in systemic lupus erythematosus. Int J Clin Rheumtol, 2012 7(2): p. 217-227. [PubMed: 22737181]

2. Omdal R, et al., Fatigue in patients with systemic lupus erythematosus: the psychosocial aspects. J Rheumatol, 2003 30(2): p. 283-7. [PubMed: 12563681]

3. Skoie IM, et al., Fatigue in psoriasis: a controlled study. Br J Dermatol, 2017 177(2): p. 505-512. [PubMed: 28182255]

4. Ingves $\mathrm{C}$ and Jemec GB, Combined imiquimod and acitretin for non-surgical treatment of basal cell carcinoma. Scand J Plast Reconstr Surg Hand Surg, 2003 37(5): p. 293-5. [PubMed: 14649688]

5. Dagfinrud H, et al., Fatigue in patients with ankylosing spondylitis: A comparison with the general population and associations with clinical and self-reported measures. Arthritis Rheum, 2005 53(1): p. 5-11. [PubMed: 15696569]

6. McHorney CA, Ware JE Jr., and Raczek AE, The MOS 36-Item Short-Form Health Survey (SF-36): II. Psychometric and clinical tests of validity in measuring physical and mental health constructs. Med Care, 1993 31(3): p. 247-63. [PubMed: 8450681]

7. Dantzer R, Cytokine, sickness behavior, and depression. Immunol Allergy Clin North Am, 2009 29(2): p. 247-64. [PubMed: 19389580]

8. Maes M, Bosmans E, and Meltzer HY, Immunoendocrine aspects of major depression. Relationships between plasma interleukin- 6 and soluble interleukin-2 receptor, prolactin and cortisol. Eur Arch Psychiatry Clin Neurosci, 1995 245(3): p. 172-8. [PubMed: 7669825]

9. Kiecolt-Glaser JK and Glaser R, Depression and immune function: central pathways to morbidity and mortality. J Psychosom Res, 2002 53(4): p. 873-6. [PubMed: 12377296]

10. Okon LG and Werth VP, Cutaneous lupus erythematosus: diagnosis and treatment. Best Pract Res Clin Rheumatol, 2013 27(3): p. 391-404. [PubMed: 24238695] 
11. Kuhn A, Ruland V, and Bonsmann G, Cutaneous lupus erythematosus: Update of therapeutic options: Part I. Journal of the American Academy of Dermatology, 2011 65(6): p. e179-e193. [PubMed: 20739095]

12. Wang H, et al., Dermatomyositis related to autoimmune thyroiditis. J Eur Acad Dermatol Venereol, 2011 25(9): p. 1085-93. [PubMed: 21118310]

13. Lukjanowicz M, Bobrowska-Snarska D, and Brzosko M, [Coexistence of hypothyroidism with polymyositis or dermatomyositis]. Ann Acad Med Stetin, 200652 Suppl 2: p. 49-55.

14. Engberg I, et al., Fatigue in the general population- associations to age, sex, socioeconomic status, physical activity, sitting time and self-rated health: the northern Sweden MONICA study 2014. BMC Public Health, 2017 17(1): p. 654. [PubMed: 28806984]

15. Dantzer R, et al., From inflammation to sickness and depression: when the immune system subjugates the brain. Nat Rev Neurosci, 2008 9(1): p. 46-56. [PubMed: 18073775] 
What's known/what's new statements:

- $\quad$ Fatigue is a well-established symptom of systemic lupus erythematosus but has not been well characterized in skin-limited autoimmune diseases.

- $\quad$ This study aims to examine fatigue in cutaneous lupus erythematosus, amyopathic dermatomyositis, and autoimmune blistering diseases. 


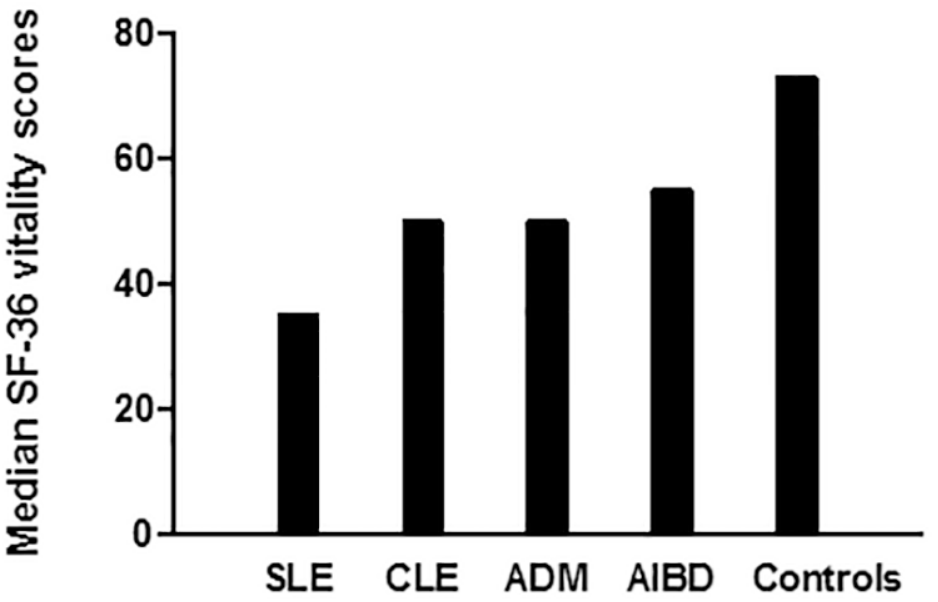

Figure 1:

Median SF-36 vitality scores between autoimmune skin disease groups and controls 


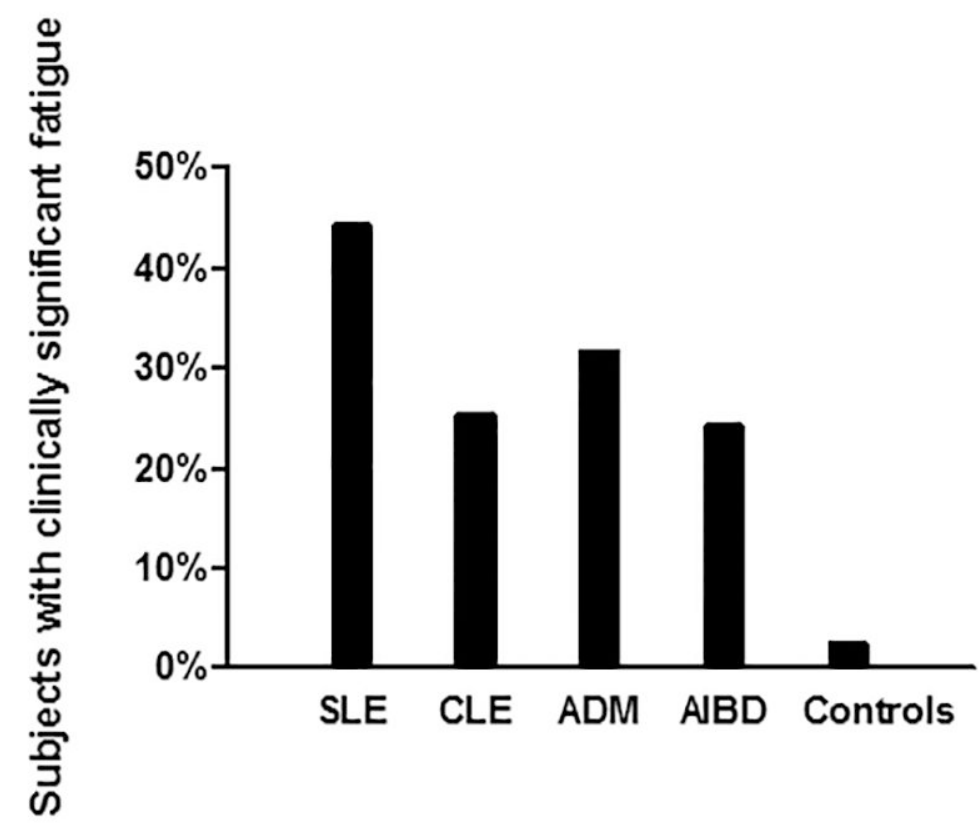

Figure 2:

Percentage of clinically significant fatigue between autoimmune skin disease groups and controls 

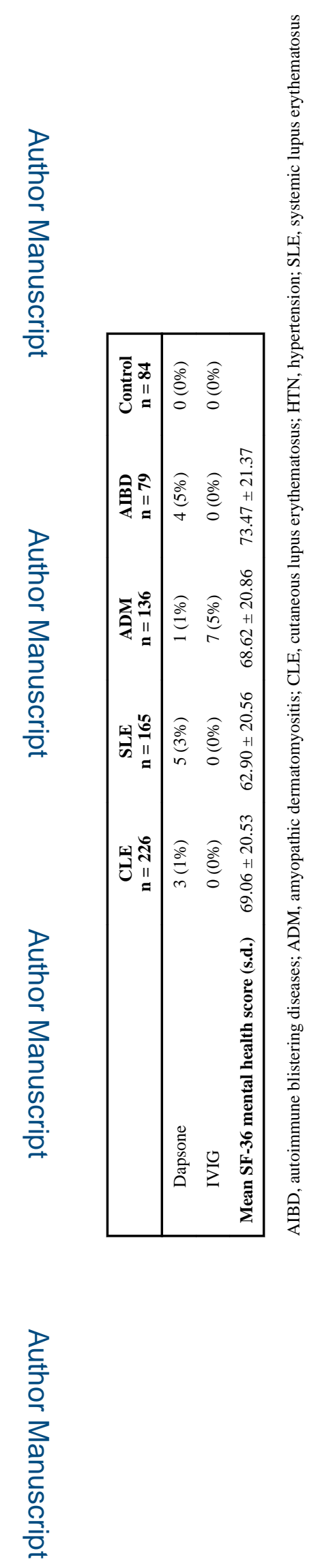

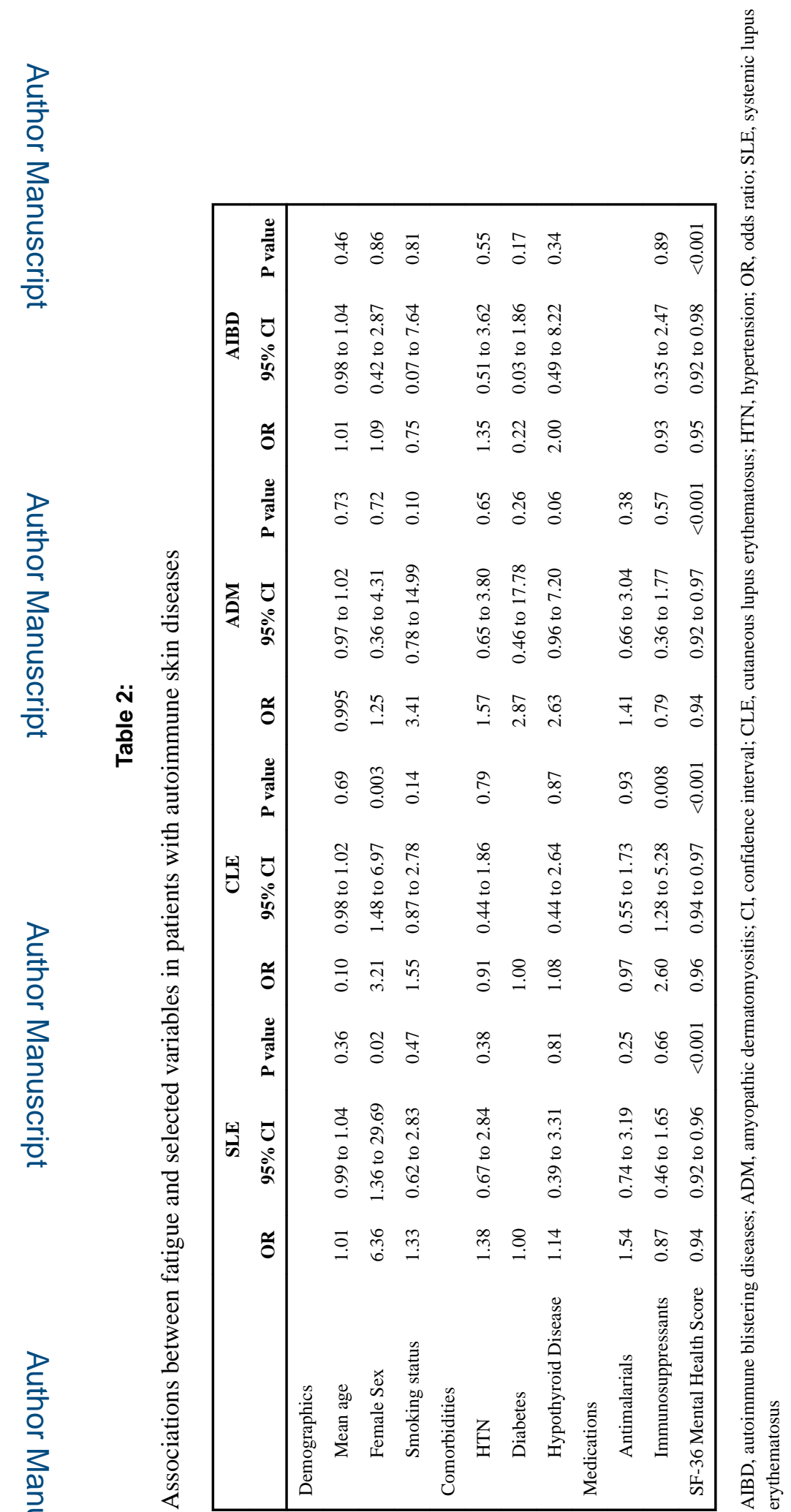

Br J Dermatol. Author manuscript; available in PMC 2020 June 01. 


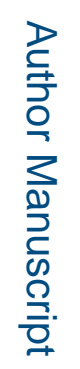

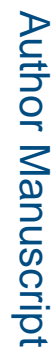

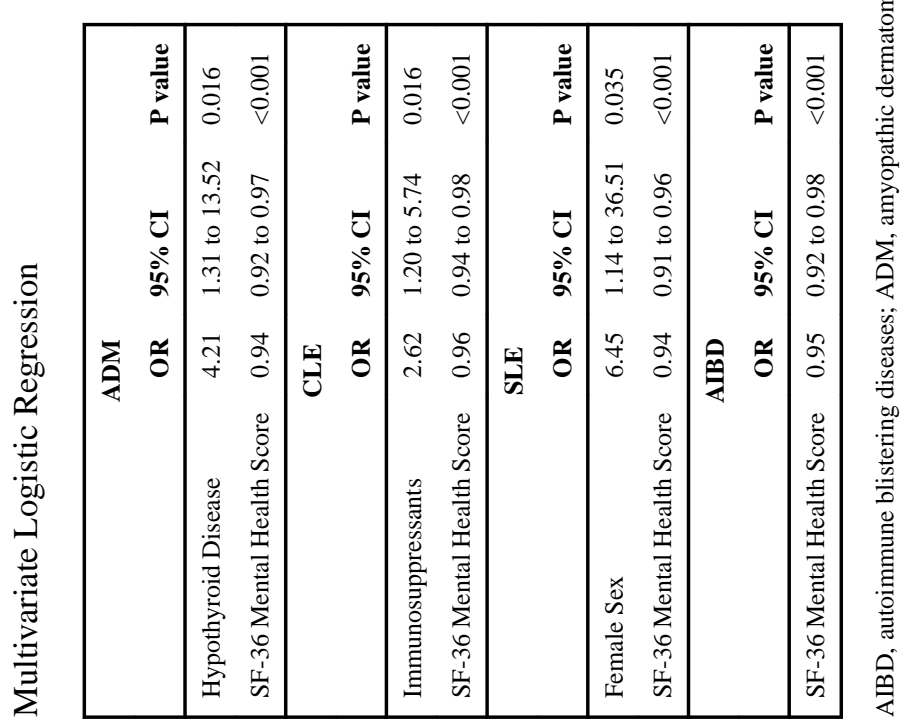

Br J Dermatol. Author manuscript; available in PMC 2020 June 01. 